KYUNGPOOK Math. J. 53(2013), 553-563

http://dx.doi.org/10.5666/KMJ.2013.53.4.553

\title{
Some Identities Involving Euler Polynomials Arising from a Non-linear Differential Equation
}

SeOG-Hoon Rim, Joohee JeOnG* And Jin-Woo PARK

Department of Mathematics Education, Kyungpook National University, Daegu, 702-701, Korea

e-mail: shrim@knu.ac.kr, jhjeong@knu.ac.kr and a0417001.paper@gmail.com

Abstract. We derive a family of non-linear differential equations from the generating functions of the Euler polynomials and study the solutions of these differential equations. Then we give some new and interesting identities and formulas for the Euler polynomials of higher order by using our non-linear differential equations.

\section{Introduction}

The Euler polynomials $E_{n}(x)$ are defined by generating functions as follows:

$$
\frac{2}{e^{t}+1} e^{x t}=\sum_{n=0}^{\infty} E_{n}(x) \frac{t^{n}}{n !} . \quad(\text { see }[2,4,5])
$$

In the special case $x=0, E_{n}(0)=E_{n}$ for $n=0,1, \ldots$ are called the $n$-th Euler numbers. (see [4])

By (1.1), we get

$$
E_{n}(x)=\sum_{\ell=0}^{\infty}\left(\begin{array}{l}
n \\
\ell
\end{array}\right) x^{n-\ell} E_{\ell}, \quad \text { for } n \in \mathbb{Z}_{+}=\mathbb{N} \cup\{0\} .
$$

Thus, by (1.1) and (1.2), we get the recursive relation for $E_{n}$ 's as follows:

$$
\begin{aligned}
& E_{0}=1, \\
& (E+1)^{n}+E_{n}= \begin{cases}2 & \text { if } n=0, \\
0 & \text { if } n>0\end{cases}
\end{aligned}
$$

* Corresponding Author.

This research was supported by Kyungpook National University Research Fund 2012.

Received November 15, 2012; accepted April 9, 2013.

2010 Mathematics Subject Classification: 05A30, $11 B 37$.

Key words and phrases: Euler polynomials, differential equation. 
with the usual convention of replacing $E^{k}$ 's in the binomial expansion of $(E+1)^{n}$ by $E_{k}$ 's.

In [11], Nielson obtained a formula for the product of two Euler polynomials $E_{n}(x)$ and $E_{m}(x)$ as follows:

$$
\begin{aligned}
E_{m}(x) E_{n}(x)= & 2 \sum_{r=1}^{m}\left(\begin{array}{c}
m \\
r
\end{array}\right) E_{r} \frac{B_{m+n-r+1}(x)}{m+n-r+1}+ \\
& +2 \sum_{s=1}^{n}\left(\begin{array}{c}
n \\
s
\end{array}\right) E_{s} \frac{B_{m+n-s+1}(x)}{m+n-s+1}+(-1)^{n+1} 2 \frac{m ! n !}{(m+n+1) !} E_{m+n+1},
\end{aligned}
$$

where $B_{m}(x)$ is the well-known Bernoulli polynomials. (See $[2,4,5,12]$ ).

For $r \in \mathbb{N}$, the Euler polynomials $E_{n}^{(r)}(x)$ of order $r$ are defined by generating functions as follows:

$$
\underbrace{\left(\frac{2}{e^{t}+1}\right) \cdots\left(\frac{2}{e^{t}+1}\right)}_{r \text {-times }} e^{x t}=\sum_{n=0}^{\infty} E_{n}^{(r)}(x) \frac{t^{n}}{n !} .
$$

In the special case $x=0, E_{n}^{(r)}(0)=E_{n}^{(r)}$ are called the $n$-th Euler numbers of order $r$. (see $[1-15,17])$

In this paper, using the idea of T. Kim [10], we derive a family of non-linear differential equations from (1.1) and study the solutions of these differential equations. Then we give some new and interesting identities and formulas for the Euler polynomials of higher order by using our non-linear differential equations.

\section{The Non-linear Differential Equations}

Throughout this paper, we put

$$
F=F(t)=\frac{1}{e^{t}+1} .
$$

Then we get

$$
F^{\prime}(t)=-\frac{e^{t}}{\left(e^{t}+1\right)^{2}}=\frac{1}{\left(e^{t}+1\right)^{2}}-\frac{1}{e^{t}+1}=F^{2}-F .
$$

We rewrite $(2.2)$ as

$$
F^{2}=F^{\prime}+F,
$$

and differentiate both sides with respect to $t$ to get

$$
2 F F^{\prime}=F^{\prime \prime}+F^{\prime} \text {. }
$$


Now, by (2.2), we may replace $F^{\prime}$ in the left hand side of (2.4) by $F^{2}-F$ to obtain

$$
2 F\left(F^{2}-F\right)=F^{\prime \prime}+F^{\prime},
$$

and consequently

$$
2 F^{3}=2 F^{2}+F^{\prime \prime}+F^{\prime} .
$$

From (2.3) and (2.6), we get

$$
\begin{aligned}
2 F^{3} & =2\left(F^{\prime}+F\right)+F^{\prime \prime}+F^{\prime} \\
& =F^{\prime \prime}+3 F^{\prime}+2 F .
\end{aligned}
$$

Now we repeat the process from (2.4) to (2.7). We start from differentiating both sides of (2.7).

(9') $3 ! F^{2} F^{\prime}=F^{(3)}+3 F^{(2)}+2 F^{(1)}$,

$\left(10^{\prime}\right) \quad 3 ! F^{2}\left(F^{2}-F\right)=F^{(3)}+3 F^{(2)}+2 F^{(1)}$,

$\left(11^{\prime}\right) \quad 3 ! F^{4}=3 ! F^{3}+\left(F^{(3)}+3 F^{(2)}+2 F^{(1)}\right)$,

$3 ! F^{4}=3\left(F^{\prime \prime}+3 F^{\prime}+2 F\right)+F^{(3)}+3 F^{(2)}+2 F^{(1)}, \quad$ from $(2.7)$ and $\left(11^{\prime}\right)$

$\left(12^{\prime}\right) \quad 3 ! F^{4}=F^{(3)}+6 F^{(2)}+11 F^{(1)}+6 F$.

We want to generalize the pattern $(2.3),(2.7)$ and $\left(12^{\prime}\right)$ to obtain

$$
(N-1) ! F^{N}=\sum_{k=0}^{N-1} a_{k}(N) F^{(k)}, \quad \text { for all } N \in \mathbb{N}
$$

for some suitable coefficients $a_{k}(N)$ 's. By letting $N=1$ in (2.8), it is easy to get

$$
a_{0}(1)=1 .
$$

Taking the derivative of (2.8), we have

$$
N ! F^{N-1} F^{\prime}=\sum_{k=0}^{N-1} a_{k}(N) F^{(k+1)}=\sum_{k=1}^{N} a_{k-1}(N) F^{(k)} .
$$

But

$$
\begin{aligned}
N ! F^{N-1} F^{\prime} & =N ! F^{N-1}\left(F^{2}-F\right) \quad(\text { by }(2.2)) \\
& =N ! F^{N+1}-N ! F^{N} .
\end{aligned}
$$

Thus

$$
\sum_{k=1}^{N} a_{k-1}(N) F^{(k)}=N ! F^{N+1}-N ! F^{N}
$$


and hence

$$
\begin{aligned}
N ! F^{N+1} & =N ! F^{N}+\sum_{k=1}^{N} a_{k-1}(N) F^{(k)} \\
& =N(N-1) ! F^{N}+\sum_{k=1}^{N} a_{k-1}(N) F^{(k)} \\
& \left.=N \sum_{k=0}^{N-1} a_{k}(N) F^{(k)}+\sum_{k=1}^{N} a_{k-1}(N) F^{(k)}, \quad \text { (by }(2.8)\right) .
\end{aligned}
$$

By replacing $N$ by $N+1,(2.8)$ may be rewritten as

$$
N ! F^{N+1}=\sum_{k=0}^{N} a_{k}(N+1) F^{(k)} .
$$

Combining (2.12) and (2.11), we get

$$
\sum_{k=0}^{N} a_{k}(N+1) F^{(k)}=N \sum_{k=0}^{N-1} a_{k}(N) F^{(k)}+\sum_{k=1}^{N} a_{k-1}(N) F^{(k)} .
$$

By comparing the coefficients of $F^{(k)}$ 's in the left and right side of above identity, we obtain the following recursive formulas:

$$
\begin{aligned}
& a_{0}(N+1)=N a_{0}(N), \\
& a_{k}(N+1)=N a_{k}(N)+a_{k-1}(N), \quad(1 \leq k \leq N-1) \\
& a_{N}(N+1)=a_{N-1}(N),
\end{aligned}
$$

where we set

$$
a_{k}(N)=0 \text { for } k \geq N \text { or } k<0 .
$$

From $(2.9),(2.14),(2.15),(2.16)$ and $(2.17)$, T. Kim was able to obtain an explicit formula for $a_{k}(N)$ as follows. (see [10])

$$
a_{k}(N)=\frac{N !}{(k+1) !} \sum_{\ell_{1}+\cdots+\ell_{k+1}=N} \frac{1}{\ell_{1} \ell_{2} \cdots \ell_{k+1}} .
$$

Therefore (2.8) now becomes

$$
(N-1) ! F^{N}=\sum_{k=0}^{N-1} \frac{N !}{(k+1) !} \sum_{\ell_{1}+\cdots+\ell_{k+1}=N} \frac{1}{\ell_{1} \ell_{2} \cdots \ell_{k+1}} F^{(k)},
$$

and consequently we obtain the following theorem. 
Theorem 2.1. For each $N \in \mathbb{N}$, the following non-linear differential equation with respect to $t$ :

$$
F^{N}(t)=N \sum_{k=0}^{N-1} \frac{1}{(k+1) !} \sum_{\ell_{1}+\cdots+\ell_{k+1}=N} \frac{1}{\ell_{1} \ell_{2} \cdots \ell_{k+1}} F^{(k)}(t)
$$

where $F^{(k)}(t)=\frac{d^{k} F(t)}{d t^{k}}$ and $F^{N}(t)=\underbrace{F(t) \times \cdots \times F(t)}_{N \text {-times }}$ has a solution

$$
F(t)=\frac{1}{e^{t}+1}
$$

\section{Some Identities Concerning Euler Polynomials of Order $r$}

We set

$$
\begin{aligned}
F^{N}(t, x) & =\underbrace{F(t) \times F(t) \times \cdots \times F(t)}_{N \text {-times }} e^{x t}, \quad \text { for } N \in \mathbb{N}, \\
F^{(k)}(t, x) & =F^{(k)}(t) e^{x t}, \quad \text { for } k \in \mathbb{Z}_{+} .
\end{aligned}
$$

Then, by multiplying $e^{x t}$ to both sides of (2.19), we have the following corollary.

Corollary 3.1. For each $n \in \mathbb{N}$, the differential equation

$$
F^{N}(t, x)=N \sum_{k=0}^{N-1} \frac{1}{(k+1) !} \sum_{\ell_{1}+\cdots+\ell_{k+1}=N} \frac{1}{\ell_{1} \ell_{2} \cdots \ell_{k+1}} F^{(k)}(t, x)
$$

has a solution $F(t, x)=\frac{e^{x t}}{e^{t}+1}$.

The goal of this section is to find an explicit formula for Euler polynomials of order $r$ using the Euler numbers. But first, we will work on Euler numbers of order $r$ instead of Euler polynomials of order $r$.

By letting $x=0$ in (1.1) and (1.4), we get

$$
\begin{aligned}
& \frac{2}{e^{t}+1}=\sum_{n=0}^{\infty} E_{n} \frac{t^{n}}{n !}, \\
& \underbrace{\left(\frac{2}{e^{t}+1}\right) \times \cdots \times\left(\frac{2}{e^{t}+1}\right)}_{N \text {-times }}=\sum_{n=0}^{\infty} E_{n}^{(N)} \frac{t^{n}}{n !} .
\end{aligned}
$$


From (2.1) and (3.4), we get

$$
F(t)=\frac{1}{2} \sum_{n=0}^{\infty} E_{n} \frac{t^{n}}{n !}
$$

and from (3.5),

$$
\begin{aligned}
F^{N}(t) & =\left(\frac{1}{e^{t}+1}\right)^{N}=\frac{1}{2^{N}}\left(\frac{2}{e^{t}+1}\right)^{N} \\
& =\frac{1}{2^{N}} \sum_{n=0}^{\infty} E_{n}^{(N)} \frac{t^{n}}{n !} .
\end{aligned}
$$

Note that we have the following by differentiating both sides of (3.6) $k$-times:

$$
F^{(k)}(t)=\frac{1}{2} \sum_{n=0}^{\infty} E_{n+k} \frac{t^{n}}{n !} .
$$

From (2.19) in Theorem 2.1, (3.7) and (3.8), we get

$$
\begin{aligned}
N \sum_{k=0}^{N-1} \frac{1}{(k+1) !} & \sum_{\ell_{1}+\cdots+\ell_{k+1}=N} \frac{1}{\ell_{1} \ell_{2} \cdots \ell_{k+1}} F^{(k)}(t) \\
= & N \sum_{k=0}^{N-1} \frac{1}{(k+1) !} \sum_{\ell_{1}+\cdots+\ell_{k+1}=N} \frac{1}{\ell_{1} \ell_{2} \cdots \ell_{k+1}} \frac{1}{2} \sum_{n=0}^{\infty} E_{n+k} \frac{t^{n}}{n !} \\
= & \frac{1}{2} N \sum_{n=0}^{\infty} \sum_{k=0}^{N-1} \frac{1}{(k+1) !} \sum_{\ell_{1}+\cdots+\ell_{k+1}=N} \frac{E_{n+k}}{\ell_{1} \ell_{2} \cdots \ell_{k+1}} \frac{t^{n}}{n !} \\
= & \frac{1}{2^{N}} \sum_{n=0}^{\infty} E_{n}^{(N)} \frac{t^{n}}{n !} .
\end{aligned}
$$

Finally, from (3.9) and (3.10) we obtain the following theorem.

Theorem 3.2. For $N \in \mathbb{N}, n \in \mathbb{Z}_{+}$, the $n$-th Euler number of order $N$ is given by

$$
E_{n}^{(N)}=N 2^{N-1} \sum_{k=0}^{N-1} \frac{1}{(k+1) !} \sum_{\ell_{1}+\cdots+\ell_{k+1}=N} \frac{E_{n+k}}{\ell_{1} \ell_{2} \cdots \ell_{k+1}} .
$$


From (3.7) and (3.4), we can derive the following equation:

$$
\begin{aligned}
\sum_{n=0}^{\infty} E_{n}^{(N)} \frac{t^{n}}{n !} & =\left(\frac{2}{e^{t}+1}\right)^{N} \\
& =\left(\sum_{\ell_{1}=0}^{\infty} E_{\ell_{1}} \frac{t^{\ell_{1}}}{\ell_{1} !}\right) \times \cdots \times\left(\sum_{\ell_{N}=0}^{\infty} E_{\ell_{N}} \frac{t^{\ell_{N}}}{\ell_{N} !}\right) \\
& =\sum_{n=0}^{\infty}\left(\sum_{\ell_{1}+\cdots+\ell_{N}=n} \frac{E_{\ell_{1}} \cdots E_{\ell_{N}} n !}{\ell_{1} ! \cdots \ell_{N} !}\right) \frac{t^{n}}{n !} \\
& =\sum_{n=0}^{\infty}\left(\begin{array}{c}
n \\
\sum_{\ell_{1}+\cdots+\ell_{N}=n}\left(\begin{array}{c}
n \\
\ell_{1} \cdots \ell_{N}
\end{array}\right) E_{\ell_{1}} \cdots E_{\ell_{N}}
\end{array}\right) \frac{t^{n}}{n !} .
\end{aligned}
$$

From (3.11) and (3.12) we get the following corollary.

\section{Corollary 3.3.}

$$
\sum_{\ell_{1}+\cdots+\ell_{N}=n}\left(\begin{array}{c}
n \\
\ell_{1} \cdots \ell_{N}
\end{array}\right) E_{\ell_{1}} \cdots E_{\ell_{N}}=N 2^{N-1} \sum_{k=0}^{N-1} \frac{1}{(k+1) !} \sum_{\ell_{1}+\cdots+\ell_{k+1}=N} \frac{E_{n+k}}{\ell_{1} \ell_{2} \cdots \ell_{k+1}} .
$$

We obtained Theorem 3.2, a formula for the $n$-th Euler numbers of order $N$, using Theorem 2.1. If we use Corollary 3.1 instead of Theorem 2.1, then we can obtain Theorem 3.4, a formula for the $n$-th Euler polynomials of order $N$.

We start from (3.2) and (3.8) as follows:

$$
\begin{aligned}
F^{(k)}(t, x) & =F^{(k)}(t) e^{x t}=\frac{1}{2} \sum_{n=0}^{\infty} E_{n+k} \frac{t^{n}}{n !} e^{x t} \\
& =\frac{1}{2} \sum_{m=0}^{\infty} E_{m+k} \frac{t^{m}}{m !} \sum_{\ell=0}^{\infty} \frac{t^{\ell}}{\ell !} x^{\ell} \\
& =\frac{1}{2} \sum_{n=0}^{\infty}\left(\sum_{m+\ell=n} \frac{n !}{m ! \ell !} \frac{t^{n}}{n !} E_{m+k} x^{\ell}\right) \\
& =\frac{1}{2} \sum_{n=0}^{\infty}\left(\sum_{m=0}^{n}\left(\begin{array}{c}
n \\
m
\end{array}\right) E_{m+k} x^{n-m}\right) \frac{t^{n}}{n !} .
\end{aligned}
$$


In Corollary 3.1 , replace $F^{(k)}(t, x)$ by (3.14) to get

$$
\begin{aligned}
& F^{N}(t, x) \\
& \quad=N \sum_{k=0}^{N-1} \frac{1}{(k+1) !} \sum_{\ell_{1}+\cdots+\ell_{k+1}=N} \frac{1}{\ell_{1} \ell_{2} \cdots \ell_{k+1}} \frac{1}{2} \sum_{n=0}^{\infty}\left(\sum_{m=0}^{n}\left(\begin{array}{c}
n \\
m
\end{array}\right) E_{m+k} x^{n-m}\right) \frac{t^{n}}{n !}
\end{aligned}
$$

$$
=\sum_{n=0}^{\infty}\left(\frac{N}{2} \sum_{k=0}^{N-1} \frac{1}{(k+1) !} \sum_{\ell_{1}+\cdots+\ell_{k+1}=N} \frac{1}{\ell_{1} \ell_{2} \cdots \ell_{k+1}} \sum_{m=0}^{n}\left(\begin{array}{c}
n \\
m
\end{array}\right) E_{m+k} x^{n-m}\right) \frac{t^{n}}{n !} .
$$

But (1.4) can be written as

$$
2^{N} F^{N}(t, x)=\sum_{n=0}^{\infty} E_{n}^{(N)}(x) \frac{t^{n}}{n !}
$$

Finally, from (3.16) and (3.17), we get the following theorem.

Theorem 3.4. For $N \in \mathbb{N}, n \in \mathbb{Z}_{+}$, the $n$-th Euler polynomial of order $N$ is given by

$$
E_{n}^{(N)}(x)=N 2^{N-1} \sum_{k=0}^{N-1} \frac{1}{(k+1) !} \sum_{\ell_{1}+\cdots+\ell_{k+1}=N} \frac{1}{\ell_{1} \ell_{2} \cdots \ell_{k+1}} \sum_{m=0}^{n}\left(\begin{array}{c}
n \\
m
\end{array}\right) E_{m+k} x^{n-m} .
$$

We will conclude our paper by giving two more formulas for $E_{n}^{(N)}(x)$. They are given in Proposition 3.5 and Proposition 3.6.

First, multiply $2^{N} e^{x t}$ to both sides of (3.7). Then we have

$$
2^{N} F^{N}(t) e^{x t}=\sum_{n=0}^{\infty} E_{n}^{(N)} \frac{t^{n}}{n !} e^{x t}
$$


Then apply the usual trick of utilizing the Taylor expansion of $e^{x t}$ to get

$$
\begin{aligned}
2^{N} F^{N}(t) e^{x t} & =\left(\sum_{\ell=0}^{\infty} E_{\ell}^{(N)} \frac{t^{\ell}}{\ell !}\right)\left(\sum_{m=0}^{\infty} x^{m} \frac{t^{m}}{m !}\right) \\
& =\sum_{n=0}^{\infty}\left(\sum_{\ell+m=n} E_{\ell}^{(N)} \frac{n !}{\ell ! m !} \frac{t^{n}}{n !} x^{m}\right) \\
& =\sum_{n=0}^{\infty}\left(\sum_{\ell=0}^{n}\left(\begin{array}{l}
n \\
\ell
\end{array}\right) x^{n-\ell} E_{\ell}^{(N)}\right) \frac{t^{n}}{n !}
\end{aligned}
$$

Note that (3.17) can be written as

$$
2^{N} F^{N}(t, x)=2^{N} F^{N}(t) e^{x t}=\sum_{n=0}^{\infty} E_{n}^{(N)}(x) \frac{t^{n}}{n !} .
$$

By comparing the coefficients of $\frac{t^{n}}{n !}$ in the r.h.s's of above two equations, we get

\section{Proposition 3.5.}

$$
E_{n}^{(N)}(x)=\sum_{\ell=0}^{n}\left(\begin{array}{l}
n \\
\ell
\end{array}\right) x^{n-\ell} E_{\ell}^{(N)}
$$

Second, rewrite (1.4) as

$$
\sum_{n=0}^{\infty} E_{n}^{(N)}(x) \frac{t^{n}}{n !}=2^{N} F^{N}(t) e^{t x}
$$

Using (3.6) and the Taylor expansion for $e^{t x}$, above equation becomes

$$
\begin{aligned}
& \sum_{n=0}^{\infty} E_{n}^{(N)}(x) \frac{t^{n}}{n !}=\left(\sum_{\ell_{1}=0}^{\infty} \frac{E_{\ell_{1}}}{\ell_{1} !} t^{\ell_{1}}\right) \times \cdots \times\left(\sum_{\ell_{1}=0}^{\infty} \frac{E_{\ell_{N}}}{\ell_{N} !} t^{\ell_{N}}\right) \sum_{m=0}^{\infty} \frac{x^{m}}{m !} t^{m} \\
& =\sum_{n=0}^{\infty}\left(\sum_{\ell 1+\cdots+\ell_{N}+m=n} \frac{E_{\ell_{1}} \cdots E_{\ell_{N}}}{\ell_{1} ! \cdots \ell_{N} ! m !} x^{m} n !\right) \frac{t^{n}}{n !} \\
& =\sum_{n=0}^{\infty}\left(\sum_{\ell 1+\cdots+\ell_{N}+m=n}\left(\begin{array}{c}
n \\
\ell_{1}, \ldots, \ell_{N}, m
\end{array}\right) E_{\ell_{1}} \cdots E_{\ell_{N}} x^{m}\right) \frac{t^{n}}{n !}
\end{aligned}
$$

By comparing the coefficients of $t^{n}$ 's on both sides, we finally get 


\section{Proposition 3.6.}

$$
E_{n}^{(N)}(x)=\sum_{\ell 1+\cdots+\ell_{N}+m=n}\left(\begin{array}{c}
n \\
\ell_{1}, \ldots, \ell_{N}, m
\end{array}\right) E_{\ell_{1}} \cdots E_{\ell_{N}} x^{m} .
$$

\section{References}

[1] I. N. Cangul and Y. Simsek, A note on interpolation functions of the Frobenius-Euler numbers, in: Application of Mathematics in Technical and Natural Sciences, in AIP Conf. Proc. 1301, Amer. Inst. Phys., Melville, NY(2010), 59-67.

[2] L. Carlitz, Eulerian numbers and polynomials, Math. Mag. 23(1959), 247-260.

[3] L. Carlitz, Note on the integral of the product of several Bernoulli polynomials, J. London Math. Soc. 34(1959), 361-363.

[4] L. Carlitz, The product of two Eulerian polynomials, Math. Mag. 36(1963), 37-41.

[5] K. W. Hwang and D. V. Dolgy and T. Kim and S.S. Lee, On the higher-order q-Euler numbers and polynomials with weight $\alpha$, Discrete Dyn. Nat. Soc. 2011 Article ID 354329, 12page(2011).

[6] L. C. Jang, A study on the distribution of twisted q-Genocchi polynomials, Adv. Stud. Contemp. Math. 18(2)(2009), 181-189.

[7] T. Kim, q-generalized Euler numbers and polynomials, Russian J. Math. Phys. 13(3)(2006), 293-298.

[8] T. Kim, Some identities on the q-Euler polynomials of higher order and q-Stirling numbers by the fermionic $p$-adic integral on $\mathbb{Z}_{p}$, Russian J. Math. Phys. 16(4)(2009), 484-491.

[9] T. Kim, New approach to q-Euler polynomials of higher order, Russian J. Math. Phys. 17(2)(2010), 218-225.

[10] T. Kim, Identities involving Frobenius-Euler polynomials arising from non-linear differential equations, J. of Number Theory, 132(2012), 2854-2865.

[11] N. Nielson, Traite elementaire des nombres de Bernoulli, Paris(1923).

[12] H. Ozden and I.N. Cangul and Y. Simsek, Multivariate interpolation functions of higher-order q-Euler numbers and their applications, Abstract and Applied Analysis 2008 Article ID 390857, 16page(2008).

[13] H. Ozden and I. N. Cangul and Y. Simsek, Remarks on sum of products of $(h, q)$ twisted Euler polynomials and numbers, J. Inequal. Appl. 2008 Article ID 816129, 8page(2008).

[14] C. S. Ryoo, Some identities of the twisted q-Euler numbers and polynomials associated with q-Bernstein polynomials, Proc. Jangjeon Math. Soc. 14(2011), 239-348.

[15] Y. Simsek, Special functions related to Dedekind-type DC-sums and their applications, Russian J. Math. Phys. 17(4)(2010), 495-508. 
[16] Y. Simsek, Complete sum of products of $(h, q)$-extension of Euler polynomials and numbers, J. Difference Equ. Appl. 16(11)(2010), 1331-1348.

[17] Y. Simsek and O. Yureki and V. Kurt, On interpolation functions of the twisted generalized Frobenbius-Euler numbers, Adv. Stud. Contemp. Math. 15(2)(2007), 187194. 